\title{
The Knowledge of Expert Opinion in Intuitionistic Fuzzy Linear Programming Problem
}

\author{
A. Nagoorgani, ${ }^{1}{ }^{\text {J. Kavikumar, }}{ }^{2}$ and K. Ponnalagu ${ }^{3}$ \\ ${ }^{1}$ PG \& Research Department of Mathematics, Jamal Mohamed College (Autonomous), Tiruchirappalli, Tamil Nadu 620 020, India \\ ${ }^{2}$ Department of Mathematics, Faculty of Science, Technology and Human Development, Universiti Tun Hussein Onn Malaysia, \\ 86400 Johor, Malaysia \\ ${ }^{3}$ Department of Mathematics, Sri Krishna Arts and Science College (Autonomous), Coimbatore, Tamil Nadu 641 008, India
}

Correspondence should be addressed to A. Nagoorgani; ganijmc@yahoo.co.in

Received 26 March 2015; Revised 30 June 2015; Accepted 2 July 2015

Academic Editor: Yan-Jun Liu

Copyright (C) 2015 A. Nagoorgani et al. This is an open access article distributed under the Creative Commons Attribution License, which permits unrestricted use, distribution, and reproduction in any medium, provided the original work is properly cited.

In real life, information available for certain situations is vague and such uncertainty is unavoidable. One possible solution is to consider the knowledge of experts on the parameters involved as intuitionistic fuzzy data. We examine a linear programming problem in which all the coefficients are intuitionistic in nature. An approach is presented to solve an intuitionistic fuzzy linear programming problem. In this proposed approach, a procedure for allocating limited resources effectively among competing demands is developed. An example is given to highlight the illustrated study.

\section{Introduction}

A linear programming problem is a mathematical program in which the objective function is linear and the constraints consist of linear equalities and linear inequalities. The first and most fruitful industrial applications of linear programming can be found in the petroleum industry, including oil extraction, refining, blending, and distribution. The computational task is then to devise an algorithm for these systems to choose the best schedule of actions from among the possible alternatives. Some people learn to make such selections via intuitive processes. In some cases, making the right selection is mainly a problem of organizing and interpreting facts. Linear programming has proved to be extremely useful for solving certain types of industrial problems for it provides a precise way of using statements of limitations such as "not more than" and "not less than" in mathematical computations. When applying OR methods to industrial problems, for instance, the problems to be modelled and solved are normally quite clear cut, well described, and crisp. They can generally be modelled and solved by using classical linear programming methods. If uncertainty occurs, it can be properly modelled using fuzzy theory.
The linear programming problems in which at least one coefficient is a fuzzy number when one or more coefficients of linear programming problems have uncertain values are known as fuzzy linear programming problems (FLPP). It is regarded to treat uncertainty of optimization problems, such as fuzzy data envelopment analysis and fuzzy network optimization $[1,2]$. The fuzzy linear programming problems in which all the parameters as well as the variables are represented by fuzzy numbers are known as fully fuzzy linear programming problems. The main advantage of fuzzy linear programming problems compared to the crisp problem formulation is the fact that the decision maker is not forced into a precise formulation. Over the past decades, solving fuzzy linear programming has become one of the fundamental research subjects in the field of fuzzy sets and systems. Fuzzy linear programming applications in real world situations are numerous and diverse.

Though fuzzy optimization formulations are more flexible, one of the poorly studied problems in this field is the definition of membership degrees. Fuzzy set theory has been widely developed and various modifications have been done. Out of several higher order fuzzy sets, intuitionistic fuzzy sets (IFS) have been found to be highly useful in dealing with 
vagueness. Here, the degrees of satisfaction and rejection are considered so that the sum of both the values is always less than or equal to one. The concept of IFS was viewed as an alternative approach for imprecise data. Therefore, considering nonmembership function as the complement of membership function developed Intuitionistic Fuzzy Optimization (IFO) problems. The main advantage of IFO problems is that they are given the richest apparatus for the formulation of optimization problems and the solution of IFO problems satisfies the objective with a higher degree of determinacy compared to the fuzzy and crisp cases. In order to avoid unrealistic modelling, the use of intuitionistic fuzzy linear programming problem (IFLPP) can be recommended. Their application implies that the problems will be solved in an interactive way. In this paper, we consider a problem in which all coefficients and variables are intuitionistic fuzzy triangular numbers in nature. In this manner, we want to propose a new matrix-analysis method to improve the efficiency of solving large-scale IFLPP, which will reduce the number of steps in the classical simplex method. The basic idea of this method is to arrange IFLPP data in matrix form and solve for various determinants to obtain the optimum solution of IFLPP. To illustrate the proposed method, numerical example is solved and the obtained result is discussed.

The paper is organized as follows. We present the works related to finding the optimal solution of an intuitionistic fuzzy linear programming problem (IFLPP) in Section 2. Section 3 provides preliminary background on intuitionistic fuzzy sets (IFS), intuitionistic fuzzy numbers (IFN), and IFLPP. The procedure for the proposed method is described in Section 4. An illustrative example is explained briefly in Section 5. Finally, conclusions are presented in Section 6.

\section{Related Works}

The research towards uncertain systems has attracted a lot of attention [3] especially the adaptive control of linear and nonlinear systems with completely unknown functions. The fuzzy logic systems (FLS), the neural networks (NN), and the fuzzy-neural networks (FNN) are very effective tools for controlling uncertainty systems [4]. As an application, the FLS, NN, and FNN have been widely used in the area of system modeling [5], fuzzy control [6], and fuzzy optimization problems [7]. Fuzzy control, which directly uses fuzzy rules, is the most important application in fuzzy theory. In a practical situation, sometimes it is quite difficult to obtain an optimal solution for fuzzy optimization problems; the use of fuzzy controls helps to find better solutions by the decision maker in order to terminate mathematical programming algorithms [8]. The intuitionistic fuzzy set theory is an extension of the fuzzy set theory by Atanassov [9] and intuitionistic fuzzy linear programming problem (IFLPP) is a special type of fuzzy linear programming problem (FLPP). There are lots of articles in this area which cannot be reviewed completely and only a few of them are reviewed here. Interval valued intuitionistic fuzzy sets were first introduced by Atanassov and Gargov [10]; since then there has been many types of intuitionistic fuzzy numbers (IFN) addressed such as interval valued intuitionistic fuzzy numbers (IVIFN), triangular intuitionistic fuzzy numbers (TIFNs), and trapezoidal intuitionistic fuzzy numbers. Mahapatra and Roy [11] discussed briefly intuitionistic fuzzy numbers and their arithmetic operations. The arithmetic operations and logic operations of triangular intuitionistic fuzzy numbers have been addressed by Wang et al. [12]. Ranking of intuitionistic fuzzy numbers plays a vital role in practical problems and so $\mathrm{Li}$ [13] developed a new ranking method based on the concept of a ratio of the index of the ambiguity index. The article by Wu and Chiclana [14] describes new score and accuracy functions for interval valued intuitionistic fuzzy numbers. A ranking procedure for triangular intuitionistic fuzzy numbers was developed by Wan and Dong [15] and its applications to multiattribute decision making was also given. Evaluation and ranking of fuzzy quantities were dealt with by Anzilli et al. [16]. The concept of the FLP was first proposed by Tanaka et al. [17], which were based on the concept of decision analysis in fuzzy environment by Bellman and Zadeh [18]. Zimmermann [19, 20] introduced fuzzy sets in operations research and presented a fuzzy approach to multiobjective linear programming problems. A new concept of the optimization problem under uncertainty was proposed and treated in [21]. On the other hand, Zhu and $\mathrm{Xu}$ [22] developed a fuzzy linear programming method to deal with group decision-making problems. The optimal solution for several degrees of feasibility of fuzzy linear and nonlinear programming problems was given by Mohtashami [23]. A real life multiobjective linear programming problem was taken into an intuitionistic fuzzy environment and solved by Nishad and Singh [24]. Moreover, Ye [25] proposed a linear programming model to solve interval valued intuitionistic multicriteria decision-making problems. Li [26] used interval valued intuitionistic fuzzy sets to capture fuzziness in linear programming.

Motivated by these articles, we proposed a study on the solutions of intuitionistic fuzzy linear programming problem (IFLPP). The classical simplex method requires much iteration to solve IFLPP. To overcome this limitation, a new matrix-analysis method is proposed in this paper. The IFLPP is represented in matrix format and various matrix operations are performed to obtain the optimum solution.

\section{Preliminaries}

In this section, the basic notations and definitions are presented. We start by defining an intuitionistic fuzzy set.

3.1. Intuitionistic Fuzzy Set (IFS). Given a fixed set $X=\left\{x_{1}\right.$, $\left.x_{2}, x_{3}, \ldots, x_{n}\right\}$, an intuitionistic fuzzy set is defined as $A=$ $\left(\left\langle x_{i}, \mu_{A}\left(x_{i}\right), v_{A}\left(x_{i}\right)\right\rangle \mid x_{i} \in X\right)$ which assigns to each element $x_{i}$ a membership degree $\mu_{A}\left(x_{i}\right)$ and a nonmembership degree $v_{A}\left(x_{i}\right)$ under the condition $0 \leq \mu_{A}\left(x_{i}\right)+v_{A}\left(x_{i}\right) \leq 1$, for all $x_{i} \in X$.

3.2. Intuitionistic Fuzzy Number (IFN). An intuitionistic fuzzy number $\widetilde{A}^{I}$ is

(i) an intuitionistic fuzzy subset of the real line; 
(ii) normal; that is, there is some $x_{0} \in \mathbb{R}$ such that $\mu_{\widetilde{A}^{I}}\left(x_{0}\right)=1, \nu_{\widetilde{A}^{I}}\left(x_{0}\right)=0 ;$

(iii) convex for the membership function $\mu_{\widetilde{A}^{I}}(x)$, that is; $\mu_{\widetilde{A}^{I}}\left(\lambda x_{1}+(1-\lambda) x_{2}\right) \geq \min \left(\mu_{\widetilde{A}^{I}}\left(x_{1}\right), \mu_{\widetilde{A}^{I}}\left(x_{2}\right)\right)$, for every $x_{1}, x_{2} \in \mathbb{R}, \lambda \in[0,1]$;

(iv) concave for the nonmembership function $\nu_{\widetilde{A}^{I}}(x)$, that is; $\nu_{\widetilde{A}^{I}}\left(\lambda x_{1}+(1-\lambda) x_{2}\right) \geq \max \left(\nu_{\widetilde{A}^{I}}\left(x_{1}\right), \nu_{\widetilde{A}^{I}}\left(x_{2}\right)\right)$, for every $x_{1}, x_{2} \in \mathbb{R}, \lambda \in[0,1]$.

3.3. Triangular Intuitionistic Fuzzy Number (TIFN). A triangular intuitionistic fuzzy number $\widetilde{A}^{I}$ is an intuitionistic fuzzy set in $\mathbb{R}$ with the following membership function $\mu_{\widetilde{A}^{I}}(x)$ and nonmembership function $\nu_{\widetilde{A}^{I}}(x)$ (Figure 1):

$$
\begin{gathered}
\mu_{\widetilde{A}^{I}}= \begin{cases}\frac{x-a_{1}}{a_{2}-a_{1}}, & a_{1} \leq x \leq a_{2}, \\
\frac{x-a_{3}}{a_{2}-a_{3}}, & a_{2} \leq x \leq a_{3}, \\
0, & \text { otherwise, }\end{cases} \\
\nu_{\widetilde{A}^{I}}= \begin{cases}\frac{a_{2}-x}{a_{2}-a_{1}^{\prime}}, & a_{1}^{\prime} \leq x \leq a_{2}, \\
\frac{x-a_{2}}{a_{3}^{\prime}-a_{2}}, & a_{2} \leq x \leq a_{3}^{\prime}, \\
1, & \text { otherwise, }\end{cases}
\end{gathered}
$$

where $a_{1}^{\prime} \leq a_{1} \leq a_{2} \leq a_{3} \leq a_{3}^{\prime}$ and $\mu_{\widetilde{A}^{I}}(x)+\nu_{\widetilde{A}^{I}} \leq 1$, or $\mu_{\widetilde{A}^{I}}(x)=\nu_{\widetilde{A}^{I}}(x), \forall x \in \mathbb{R}$. This TIFN is denoted by

$$
\begin{aligned}
\widetilde{A}^{I} & =\left(a_{1}, a_{2}, a_{3} ; a_{1}^{\prime}, a_{2}, a_{3}^{\prime}\right) \\
& =\left\{\left(a_{1}, a_{2}, a_{3}\right) ;\left(a_{1}^{\prime}, a_{2}, a_{3}^{\prime}\right)\right\} .
\end{aligned}
$$

3.4. Arithmetic Operations. Arithmetic operations of triangular intuitionistic fuzzy number based on $(\alpha, \beta)$-cuts method are as follows:

(i) If $\widetilde{A}^{I}=\left\{\left(a_{1}, a_{2}, a_{3}\right) ;\left(a_{1}^{\prime}, a_{2}, a_{3}^{\prime}\right)\right\}$ and $\widetilde{B}^{I}=\left\{\left(b_{1}, b_{2}, b_{3}\right)\right.$; $\left.\left(b_{1}^{\prime}, b_{2}, b_{3}^{\prime}\right)\right\}$ are two TIFNs, then their sum is $\widetilde{A}^{I}+\widetilde{B}^{I}=$ $\left\{\left(a_{1}+b_{1}, a_{2}+b_{2}, a_{3}+b_{3}\right) ;\left(a_{1}^{\prime}+b_{1}^{\prime}, a_{2}+b_{2}, a_{3}^{\prime}+b_{3}^{\prime}\right)\right\}$ which is also a TIFN.

(ii) If $\widetilde{A}^{I}=\left\{\left(a_{1}, a_{2}, a_{3}\right) ;\left(a_{1}^{\prime}, a_{2}, a_{3}^{\prime}\right)\right\}$ and $\widetilde{B}^{I}=\left\{\left(b_{1}, b_{2}, b_{3}\right)\right.$; $\left.\left(b_{1}^{\prime}, b_{2}, b_{3}^{\prime}\right)\right\}$ are two TIFNs, then their difference is $\widetilde{A}^{I}-$ $\widetilde{B}^{I}=\left\{\left(a_{1}-b_{3}, a_{2}-b_{2}, a_{3}-b_{1}\right) ;\left(a_{1}^{\prime}-b_{3}^{\prime}, a_{2}-b_{2}, a_{3}^{\prime}-b_{1}^{\prime}\right)\right\}$ which is also a TIFN.

(iii) If $\widetilde{A}^{I}=\left\{\left(a_{1}, a_{2}, a_{3}\right) ;\left(a_{1}^{\prime}, a_{2}, a_{3}^{\prime}\right)\right\}$ and $\widetilde{B}^{I}=\left\{\left(b_{1}, b_{2}, b_{3}\right)\right.$; $\left.\left(b_{1}^{\prime}, b_{2}, b_{3}^{\prime}\right)\right\}$ are two TIFNs, then their product is $\widetilde{A}^{I} \times$ $\widetilde{B}^{I}=\left\{\left(a_{1} b_{1}, a_{2} b_{2}, a_{3} b_{3}\right) ;\left(a_{1}^{\prime} b_{1}^{\prime}, a_{2} b_{2}, a_{3}^{\prime} b_{3}^{\prime}\right)\right\}$ which is also a TIFN.

(iv) If $\widetilde{A}^{I}=\left\{\left(a_{1}, a_{2}, a_{3}\right) ;\left(a_{1}^{\prime}, a_{2}, a_{3}^{\prime}\right)\right\}$ is a TIFN and $y=$ $k a$ (with $k>0$ ) then $\tilde{y}^{I}=k \widetilde{A}^{I}=\left\{\left(k a_{1}, k a_{2}, k a_{3}\right)\right.$; $\left.\left(k a_{1}^{\prime}, k a_{2}, k a_{3}^{\prime}\right)\right\}$ is also a TIFN.

(v) If $\widetilde{A}^{I}=\left\{\left(a_{1}, a_{2}, a_{3}\right) ;\left(a_{1}^{\prime}, a_{2}, a_{3}^{\prime}\right)\right\}$ is a TIFN and $y=$ $k a$ (with $k<0)$ then $\widetilde{y}^{I}=k \widetilde{A}^{I}=\left\{\left(k a_{3}, k a_{2}, k a_{1}\right)\right.$; $\left.\left(k a_{3}^{\prime}, k a_{2}, k a_{1}^{\prime}\right)\right\}$ is also a TIFN.

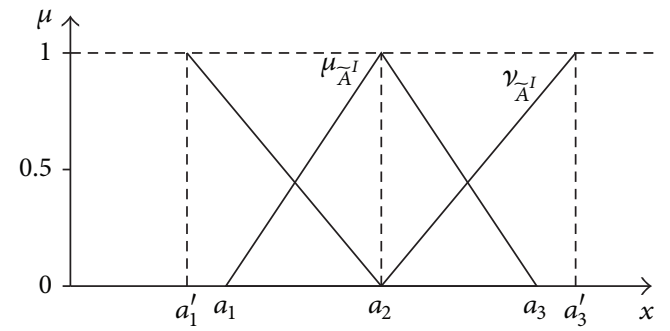

FIgURE 1: Membership and nonmembership functions of TIFN.

(vi) If $\widetilde{A}^{I}=\left\{\left(a_{1}, a_{2}, a_{3}\right) ;\left(a_{1}^{\prime}, a_{2}, a_{3}^{\prime}\right)\right\}$ and $\widetilde{B}^{I}=\left\{\left(b_{1}, b_{2}, b_{3}\right)\right.$; $\left.\left(b_{1}^{\prime}, b_{2}, b_{3}^{\prime}\right)\right\}$ are two positive TIFNs, then $\widetilde{A}^{I} / \widetilde{B}^{I}$ is also a TIFN, where $\widetilde{A}^{I} / \widetilde{B}^{I}=\left\{\left(a_{1} / b_{3}, a_{2} / b_{2}, a_{3} / b_{1}\right)\right.$; $\left.\left(a_{1}^{\prime} / b_{3}^{\prime}, a_{2} / b_{2}, a_{3}^{\prime} / b_{1}^{\prime}\right)\right\}$.

3.5. Score Function and Accuracy Function. Let $\widetilde{A}^{I}=\left\{\left(a_{1}\right.\right.$, $\left.\left.a_{2}, a_{3}\right) ;\left(a_{1}^{\prime}, a_{2}, a_{3}^{\prime}\right)\right\}$ be a TIFN; then we define a score function for membership and nonmembership as follows:

$$
\begin{aligned}
& S\left(\widetilde{A}^{I \alpha}\right)=\frac{a_{1}+2 a_{2}+a_{3}}{4}, \\
& S\left(\widetilde{B}^{I \beta}\right)=\frac{a_{1}^{\prime}+2 a_{2}+a_{3}^{\prime}}{4} .
\end{aligned}
$$

Let $\widetilde{A}^{I}=\left\{\left(a_{1}, a_{2}, a_{3}\right) ;\left(a_{1}^{\prime}, a_{2}, a_{3}^{\prime}\right)\right\}$ be a TIFN; then

$$
H\left(\widetilde{A}^{I}\right)=\frac{\left(a_{1}+2 a_{2}+a_{3}\right)+\left(a_{1}^{\prime}+2 a_{2}+a_{3}^{\prime}\right)}{8}
$$

is an accuracy function of $\widetilde{A}^{I}$, which is used to defuzzify the given number.

3.6. Ranking Using Score Function. Let $\widetilde{A}^{I}=\left\{\left(a_{1}, a_{2}, a_{3}\right) ;\left(a_{1}^{\prime}\right.\right.$, $\left.\left.a_{2}, a_{3}^{\prime}\right)\right\}$ and $\widetilde{B}^{I}=\left\{\left(b_{1}, b_{2}, b_{3}\right) ;\left(b_{1}^{\prime}, b_{2}, b_{3}^{\prime}\right)\right\}$ be two TIFNs. Let $\left(S\left(\widetilde{A}^{I \alpha}\right), S\left(\widetilde{A}^{I \beta}\right)\right)$ and $\left(S\left(\widetilde{B}^{I \alpha}\right), S\left(\widetilde{B}^{I \beta}\right)\right)$ be the scores of $\widetilde{A}^{I}$ and $\widetilde{B}^{I}$, respectively. Then consider the following:

(i) If $S\left(\widetilde{A}^{I \alpha}\right) \leq S\left(\widetilde{B}^{I \alpha}\right)$ and $S\left(\widetilde{A}^{I \beta}\right) \leq S\left(\widetilde{B}^{I \beta}\right)$, then $\widetilde{A}^{I}<\widetilde{B}^{I}$.

(ii) If $S\left(\widetilde{A}^{I \alpha}\right) \geq S\left(\widetilde{B}^{I \alpha}\right)$ and $S\left(\widetilde{A}^{I \beta}\right) \geq S\left(\widetilde{B}^{I \beta}\right)$, then $\widetilde{A}^{I}>\widetilde{B}^{I}$.

(iii) If $S\left(\widetilde{A}^{I \alpha}\right)=S\left(\widetilde{B}^{I \alpha}\right)$ and $S\left(\widetilde{A}^{I \beta}\right)=S\left(\widetilde{B}^{I \beta}\right)$, then $\widetilde{A}^{I}=\widetilde{B}^{I}$.

3.7. Intuitionistic Fuzzy Linear Programming Problem (IFLPP). A linear programming with triangular intuitionistic fuzzy variables is defined as

$$
\begin{array}{ll}
\text { (IFLP) } \max & \widetilde{Z}^{I}=\sum_{j=1}^{n} \widetilde{c}_{j}^{I} \tilde{x}_{j}^{I}, \\
\text { Subject to } & \sum_{j=1}^{n} \tilde{a}_{i j}^{I} \tilde{x}_{j}^{I} \leq \widetilde{b}_{i}^{I}, \\
& \tilde{x}_{j}^{I} \geq 0, \quad i=1,2, \ldots, m,
\end{array}
$$


where $\widetilde{A}^{I}=\left(\widetilde{a}_{i j}^{I}\right), \widetilde{c}_{j}^{I}, \widetilde{b}_{i}^{I}$, and $\tilde{x}_{j}^{I}$ are $(m \times n),(1 \times n),(m \times 1)$, and $(n \times 1)$ intuitionistic fuzzy matrices consisting of triangular intuitionistic fuzzy numbers (TIFNs).

3.8. Representation of IFLPP in Matrix Form. Consider the IFLPP defined in Section 3.7. The matrix notation of IFLPP is defined as follows:

$$
\left(\begin{array}{cccccc}
\tilde{x}_{1}^{I} & \tilde{x}_{2}^{I} & \tilde{x}_{3}^{I} & \ldots & \tilde{x}_{n}^{I} & \\
\tilde{a}_{11}^{I} & \tilde{c}_{2}^{I} & \tilde{c}_{3}^{I} & \ldots & \tilde{c}_{n}^{I} & \tilde{b}_{i}^{I} \\
\tilde{a}_{21}^{I} & \tilde{a}_{13}^{I} & \ldots & \tilde{a}_{1 n} & \tilde{b}_{1}^{I} \\
\vdots & \tilde{a}_{23}^{I} & \ldots & \tilde{a}_{2 n} & \tilde{b}_{2}^{I} \\
\tilde{a}_{m 1}^{I} & \tilde{a}_{m 2}^{I} & \tilde{a}_{m 3}^{I} & \cdots & \tilde{a}_{m n} & \tilde{b}_{m}^{I}
\end{array}\right) .
$$

3.9. Intuitionistic Fuzzy Basic Solution. A basic solution to (6) is a solution obtained by setting any $n$ intuitionistic fuzzy variables (among $m+n$ variables) equal to zero and solving remaining $m$ intuitionistic fuzzy variables provided the determinant of the coefficient of these $m$ intuitionistic fuzzy variables is nonzero. Such $m$ intuitionistic fuzzy variables (any of them may be zero) are called basic variables and remaining $n$ zero variables are called nonbasic variables. The number of intuitionistic fuzzy basic solutions obtained will be at most $(m+n) C_{m}=(m+n) ! / n ! m !$, which is the number of combinations of $n+m$ things taken $m$ at a time.

3.10. Intuitionistic Fuzzy Basic Feasible Solution. An intuitionistic fuzzy basic feasible solution is an intuitionistic fuzzy basic solution, which also satisfies (7); that is, all the basic variables are nonnegative.

3.11. Intuitionistic Fuzzy Optimum Feasible Solution. Let $\widetilde{X}^{I}=$ $\left(\tilde{x}_{1}^{I}, \tilde{x}_{2}^{I}, \tilde{x}_{3}^{I}, \ldots, \tilde{x}_{n}^{I}\right)$ be the set of all intuitionistic fuzzy feasible solutions of (5). An intuitionistic fuzzy feasible solution $\widetilde{x}_{0}^{I} \in$ $\widetilde{X}^{I}$ is said to be an intuitionistic fuzzy optimum solution to (5), if

$$
\widetilde{c}^{I} \tilde{x}_{0}^{I} \geq \widetilde{c}^{I} \tilde{x}^{I} \quad \forall \widetilde{x}^{I} \in \widetilde{X}^{I},
$$

where $\widetilde{c}^{I}=\left(\widetilde{c}_{1}^{I}, \widetilde{c}_{2}^{I}, \widetilde{c}_{3}^{I}, \ldots, \widetilde{c}_{n}^{I}\right)$ and $\tilde{c}^{I} \tilde{x}^{I}=\widetilde{c}_{1}^{I} \tilde{x}_{1}^{I}+\widetilde{c}_{2}^{I} \tilde{x}_{2}^{I}+\widetilde{c}_{3}^{I} \tilde{x}_{3}^{I}+$ $\cdots+\widetilde{c}_{n}^{I} \tilde{x}_{n}^{I}$.

Theorem 1. The set of all feasible solutions to the intuitionistic fuzzy linear programming problem is a convex set.

Proof. We need to show that every convex combination of any two feasible solutions is also a feasible solution. The theorem is true, if the set has only one element. Assume that there are at least two solutions such as $\widetilde{X}_{1}^{I}=\left(\tilde{x}_{1}^{I}, \tilde{x}_{2}^{I}, \tilde{x}_{3}^{I}, \ldots, \tilde{x}_{n}^{I}\right)$ and $\widetilde{X}_{2}^{I}=\left(\tilde{y}_{1}^{I}, \widetilde{y}_{2}^{I}, \tilde{y}_{3}^{I}, \ldots, \tilde{y}_{n}^{I}\right)$. Consider the system defined in (6) as $\widetilde{A}^{I} \widetilde{X}^{I} \leq \widetilde{b}^{I}$. Since $\widetilde{X}_{1}^{I}$ and $\widetilde{X}_{2}^{I}$ are solutions we have $\widetilde{A}^{I} \widetilde{X}_{1}^{I}=$ $\widetilde{b}^{I}$, for $\widetilde{X}_{1}^{I} \geq 0$, and $\widetilde{A}^{I} \widetilde{X}_{2}^{I}=\widetilde{b}^{I}$, for $\widetilde{X}_{2}^{I} \geq 0$. For $0 \leq \alpha \leq 1$, let $\widetilde{X}^{I}=\alpha \widetilde{X}_{1}^{I}+(1-\alpha) \widetilde{X}_{2}^{I}$ be any convex combination of $\widetilde{X}_{1}^{I}$ and $\widetilde{X}_{2}^{I}$. We note that all the elements of the vector $\widetilde{X}^{I}$ are nonnegative. Now $\widetilde{A}^{I} \widetilde{X}^{I}=\widetilde{A}^{I}\left(\alpha \widetilde{X}_{1}^{I}+(1-\alpha) \widetilde{X}_{2}^{I}\right)=\widetilde{A}^{I} \alpha \widetilde{X}_{1}^{I}+$ $\widetilde{A}^{I} \widetilde{X}_{2}^{I}-\widetilde{A}^{I} \alpha \widetilde{X}_{2}^{I}=\alpha \widetilde{b}^{I}+\widetilde{b}^{I}-\alpha \widetilde{b}^{I}=\widetilde{b}^{I}$. This completes the proof.

\section{Methodology}

This matrix-analysis method is the development of an idea or an approach to programming. It changes the form of the problem information in such a way so as to make possible direct logical comparisons of alternatives. This greatly simplifies calculation.

4.1. Steps for Setting Up and Solving a Problem. The use of matrix-analysis method involves a number of steps which follow a definite pattern. The steps in the order in which they should be taken are given below.

4.1.1. Step 1: Set Up the Unknowns. The unknowns are the amounts of each product to be made from the available amount of productive capacity to maximize profits. The unknowns represent the answers that are wanted. They will have either positive or zero values.

4.1.2. Step 2: Form a Matrix for Analysis. The first step is to arrange the problem data in a table or matrix to facilitate solution, which is a matrix consisting of the coefficients of a family of related equations and inequations. The matrix is constructed as defined in Section 3.8.

4.1.3. Step 3: Unitize the Rows of the Matrix. The purpose of this step is to set up each resource as unity. This is done by dividing the numbers in each row by their constant value at the end of the row. When this process is completed, the time to make one unit of a product is expressed as a fraction of the capacity. This calculation does not affect the profit per unit for each product. These computations in no way change the validity of the inequations in the first matrix. They state the same fact in different ways. The unitized matrix provides a way of comparing the requirements for each product in terms of the capacity of each department.

4.1.4. Step 4: Equalize the Columns of the Matrix. Since the objective is to earn as much as profit as possible, the profit possibilities of each product must be set up and compared. The process of setting up and converting the information to a comparable basis is termed as equalizing. The comparisons of profit are accomplished by establishing groups of each product selected so that the profit on each group is the same as that of a group of any other product. Therefore, we multiply each column by the least common multiple of the profits, and the matrix is now said to be a unitized, equalized matrix. However, now that direct comparison is possible, facts can be found and compared easily compared to the first matrix.

4.1.5. Step 5: Find the First Key Number. The next step is to determine whether it is more profitable to make a combination of products instead of just one product. First, 
select the largest number in each column. Secondly, select the smallest of these largest numbers. Hereafter, the smallest of the largest number is known as the first key number. The row and the column in which this number is found will be known, respectively, as the first key row and the first key column. There may be other key numbers in the matrix. If so, they will be called the second key number and the third key number. The rows and columns of the second and the third key numbers, if any, will be called the second and the third key rows and key columns, respectively.

4.1.6. Step 6: Rearrange the Matrix Based on Key Number. The sequence of the rows and columns are changed, but each number is kept in its row and column. The first key number is put in the upper left hand corner of the matrix. This immediately establishes the restriction for the first row and for the first column. The second key number is placed one row down and one column to the right. There is a requirement to be met in this arrangement; that is, the numbers to the immediate right of each key number must be smaller than the key number. The next step in the procedure is to see whether any other key number can be added to this group of figures. This can be done as it was done before.

4.1.7. Step 7: Check for Profit Potential. If $m$ constraints and $n$ unknowns are given in Step 1, consider $n$ equations with $n$ unknowns from the rearranged matrix. Solve this system of $n \times n$ equations and the solution for this system of equations gives the optimum result.

4.1.8. Step 8: Set Up a Profit Table. This step is the interpretation of the results of the previous steps. The equalized groups can be converted to actual number of units by multiplying their respective factors of least common multiples, which was considered in Step 4. The development of a profit table would be used as a guide for management decisions.

\section{Numerical Example}

A construction site engineer has been assigned the task of moving huge debris onto a field from the site. The site engineer has been informed that only a maximum of $\widetilde{100}^{I}$ vehicles are allowed, each to be used for a single round trip. There are two types of vehicles available to the site engineer, a Caterpillar articulated truck that can carry $\widetilde{24}^{I}$ cubic meters and a Howo dump truck that can carry $\widetilde{16}^{I}$ cubic meters. The articulated truck is estimated to use $\widetilde{50}^{I} \mathrm{~L}$ of fuel on a round trip, whereas the dump truck will use only $\widetilde{25}^{I} \mathrm{~L}$ of fuel per round trip. The project is granted with a total of $\widetilde{4000}^{I} \mathrm{~L}$ of fuel. The vehicles will require maintenance after each round trip: each dump truck requires $\widetilde{3}^{I} \mathrm{hrs}$ and each articulated truck requires $\widetilde{9}^{I}$ hrs. The maintenance wing has $\widetilde{720}^{I}$ hrs available. To move a maximum amount of cubic meters, what mix of articulated trucks and dump trucks should the site engineer choose?
The data is given as triangular intuitionistic fuzzy numbers as follows:

$$
\begin{aligned}
\widetilde{100}^{I} & =\{(99,100,101) ;(95,100,102)\} ; \\
\widetilde{24}^{I} & =\{(23,24,25) ;(22,24,26)\} ; \\
\widetilde{16}^{I} & =\{(15,16,17) ;(14,16,18)\} ; \\
\widetilde{50}^{I} & =\{(48,50,51) ;(47,50,52)\} ; \\
\widetilde{25}^{I} & =\{(24,25,26) ;(23,25,28)\} ; \\
\widetilde{3}^{I} & =\{(2,3,4) ;(1,3,5)\} ; \\
\widetilde{720}^{I} & =\{(715,720,723) ;(711,720,725)\} ; \\
\widetilde{9}^{I} & =\{(8,9,10) ;(7,9,11)\} ; \\
\widetilde{1}^{I} & =\{(0.5,1,1.5) ;(0.4,1,1.8)\} ; \\
\widetilde{1}^{I} & =\{(0.9,1,1.1) ;(0.8,1,1.2)\} ; \\
\widetilde{4000}^{I} & =\{(3995,4000,4010) ;(3990,4000,4015)\} .
\end{aligned}
$$

\section{Solution:}

Let $\widetilde{x}_{1}^{I}$ be the number of articulated trucks to be used.

Let $\widetilde{x}_{2}^{I}$ be the number of dump trucks to be used.

The objective function is to maximize the volume transported.

Mathematical formulation of the given problem is as follows:

$$
\operatorname{Max} \widetilde{z}^{I}=\widetilde{24}^{I} \widetilde{x}_{1}^{I}+\widetilde{16}^{I} \tilde{x}_{2}^{I},
$$

subject to the following constraints:
(A) fuel limit: $\widetilde{50}^{I} \widetilde{x}_{1}^{I}+\widetilde{25}^{I} \widetilde{x}_{2}^{I} \leq \widetilde{4000}^{I}$;
(B) maintenance hour limit: $\widetilde{3}^{I} \tilde{x}_{1}^{I}+\widetilde{9}^{I} \widetilde{x}_{2}^{I} \leq \widetilde{720}^{I}$;
(C) number limit on vehicles: $\widetilde{1}^{I} \widetilde{x}_{1}^{I}+\widetilde{1}^{I} \widetilde{x}_{2}^{I} \leq \widetilde{100}^{I}$; non-negativity of variables: $\widetilde{x}_{1}^{I}, \widetilde{x}_{2}^{I} \geq 0$,

where

$$
\begin{aligned}
& \widetilde{c}_{1}^{I}=\widetilde{24}^{I}=\{(23,24,25) ;(22,24,26)\} ; \\
& \widetilde{c}_{2}^{I}=\widetilde{16}^{I}=\{(15,16,17) ;(14,16,18)\} ; \\
& \widetilde{a}_{11}^{I}=\widetilde{50}^{I}=\{(48,50,51) ;(47,50,52)\} ; \\
& \widetilde{a}_{12}^{I}=\widetilde{25}^{I}=\{(24,25,26) ;(23,25,28)\} ;
\end{aligned}
$$




$$
\begin{aligned}
\tilde{a}_{21}^{I} & =\widetilde{3}^{I}=\{(2,3,4) ;(1,3,5)\} ; \\
\tilde{a}_{22}^{I} & =\widetilde{9}^{I}=\{(8,9,10) ;(7,9,11)\} ; \\
\tilde{a}_{31}^{I} & =\widetilde{1}^{I}=\{(0.5,1,1.5) ;(0.4,1,1.8)\} ; \\
\tilde{a}_{32}^{I} & =\widetilde{1}^{I}=\{(0.9,1,1.1) ;(0.8,1,1.2)\} ; \\
\widetilde{b}_{1}^{I} & =\widetilde{4000}^{I} \\
& =\{(3995,4000,4010) ;(3990,4000,4015)\} ; \\
\widetilde{b}_{2}^{I} & =\widetilde{720}^{I}=\{(715,720,723) ;(711,720,725)\} ; \\
\widetilde{b}_{3}^{I} & =\widetilde{100}^{I}=\{(99,100,101) ;(95,100,102)\} .
\end{aligned}
$$

Step 1. Arrange the elements in a matrix form as follows:

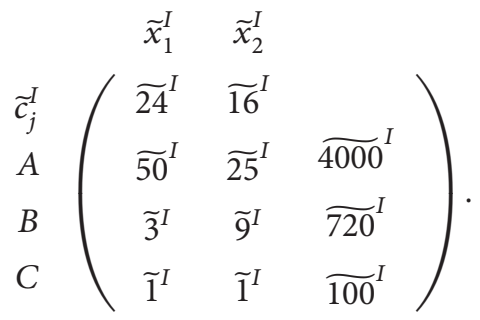

Step 2. Unitize the rows of the matrix.

Divide the numbers in each row by their constant value at the end of the row:

$$
\begin{gathered}
\widetilde{c}_{j}^{I} \\
A \\
B \\
C
\end{gathered}\left(\begin{array}{ccc}
\widetilde{x}_{1}^{I} & \tilde{x}_{2}^{I} & \\
\widetilde{0.0125}^{I} & \widetilde{0.0063}^{I} & \widetilde{1}^{I} \\
\widetilde{0.0042}^{I} & \widetilde{0.0125}^{I} & \widetilde{1}^{I} \\
\widetilde{0.01}^{I} & \widetilde{0.01}^{I} & \widetilde{1}^{I}
\end{array}\right) .
$$

The elements of first row are

$$
\begin{aligned}
& \tilde{a}_{11}^{I}=\frac{\widetilde{50}^{I}}{\widetilde{4000}^{I}}=\widetilde{0.0125}^{I}=\{(0.0119,0.0125,0.0127) ; \\
& (0.0117,0.0125,0.0130)\} ; \\
& \tilde{a}_{12}^{I}=\frac{\widetilde{25}^{I}}{\widetilde{4000}^{I}}=\widetilde{0.0063}^{I}=\{(0.0059,0.0063,0.0065) ; \\
& (0.0057,0.0063,0.0070)\} ; \\
& \tilde{a}_{13}^{I}=\frac{\widetilde{4000}^{I}}{\widetilde{4000}^{I}}=\widetilde{1}^{I}=\{(0.9963,1,1.0037) ; \\
& (0.9937,1,1.0062)\} .
\end{aligned}
$$

The elements of second row are

$$
\begin{aligned}
& \tilde{a}_{21}^{I}=\frac{\widetilde{3}^{I}}{\widetilde{720}^{I}}=\widetilde{0.0042}^{I}=\{(0.0027,0.0042,0.0055) ; \\
& (0.0013,0.0042,0.0070)\} ; \\
& \tilde{a}_{22}^{I}=\frac{\widetilde{9}^{I}}{\widetilde{720}^{I}}=\widetilde{0.0125}^{I}=\{(0.0110,0.0125,0.0139) ; \\
& (0.0096,0.0125,0.0154)\} ; \\
& \tilde{a}_{23}^{I}=\frac{\widetilde{720}^{I}}{\widetilde{720}^{I}}=\widetilde{1}^{I}=\{(0.9889,1,1.0111) ; \\
& (0.9806,1,1.0196)\} .
\end{aligned}
$$

The elements of third row are

$$
\begin{aligned}
\tilde{a}_{31}^{I} & =\frac{\widetilde{1}^{I}}{\widetilde{100}^{I}}=\widetilde{0.01}^{I} \\
& =\{(0.0049,0.01,0.0151) ;(0.0039,0.01,0.0189)\} ; \\
\tilde{a}_{32}^{I} & =\frac{\widetilde{1}^{I}}{\widetilde{100}^{I}}=\widetilde{0.01}^{I} \\
& =\{(0.0089,0.01,0.0111) ;(0.0078,0.01,0.0126)\} ; \\
\tilde{a}_{33}^{I} & =\frac{\widetilde{100}^{I}}{\widetilde{100}^{I}}=\widetilde{1}^{I} \\
& =\{(0.9801,1,1.0202) ;(0.9313,1,1.0736)\} .
\end{aligned}
$$

Step 3. Equalize the columns of the matrix.

Multiply second column by 1.5 :

$$
\begin{aligned}
& \tilde{x}_{1}^{I} \quad \tilde{x}_{2}^{I} \\
& \begin{array}{l}
\widetilde{c}_{j}^{I} \\
A \\
B \\
C
\end{array}\left(\begin{array}{ccc}
\widetilde{24}^{I} & \widetilde{24}^{I} & \\
\widetilde{0.0125}^{I} & \widetilde{0.0094}^{I} & \widetilde{1}^{I} \\
\widetilde{0.0042}^{I} & \widetilde{0.0187}^{I} & \widetilde{1}^{I} \\
\widetilde{0.01}^{I} & \widetilde{0.015}^{I} & \widetilde{1}^{I}
\end{array}\right) .
\end{aligned}
$$

The elements of the second column are

$$
\begin{aligned}
& \widetilde{24}^{I}=\{(22.5,24,25.5) ;(21,24,27)\} ; \\
& \tilde{a}_{12}^{I}=\widetilde{0.0094}^{I}=\{(0.0088,0.0094,0.0097) ; \\
& \quad(0.0085,0.0094,0.0105)\} ; \\
& \tilde{a}_{22}^{I}=\widetilde{0.0187}^{I}=\{(0.0165,0.0187,0.0208) ; \\
& \quad(0.0144,0.0187,0.0231)\} ; \\
& \tilde{a}_{32}^{I}=\widetilde{0.015}^{I}=\{(0.0133,0.015,0.0166) ; \\
& \quad(0.0117,0.015,0.0189)\} .
\end{aligned}
$$


TABLE 1

\begin{tabular}{|c|c|c|c|c|}
\hline \multirow{2}{*}{ Departments } & \multirow{2}{*}{ Availability } & \multicolumn{3}{|c|}{ Maximum quantity utilized by vehicles } \\
\hline & & Articulated truck $\tilde{x}_{1}^{I}$ & Dump truck $\tilde{x}_{2}^{I}$ & Total \\
\hline Fuel & $\widetilde{4000} \mathrm{~L}$ & $\widetilde{3000}^{L} \mathrm{~L}$ & $\widetilde{1000}^{L} \mathrm{~L}$ & $\widetilde{4000}^{1} \mathrm{~L}$ \\
\hline Maintenance hours & $\widetilde{720}^{I} \mathrm{hrs}$ & $\widetilde{180}^{I} \mathrm{hrs}$ & $\widetilde{360}^{I} \mathrm{hrs}$ & $\widetilde{540}^{I} \mathrm{hrs}$ \\
\hline Number of vehicles & $\widetilde{100}^{I}$ & $\widetilde{60}^{I}$ & $\widetilde{40}^{I}$ & $\widetilde{100}^{I}$ \\
\hline \multicolumn{2}{|c|}{ Equalized profit units } & $\widetilde{59.68}^{I}$ & $\widetilde{26.88}^{I}$ & \\
\hline \multicolumn{2}{|c|}{ Actual units produced } & $\widetilde{60}^{I}$ & $\widetilde{40}^{I}$ & $\widetilde{2080}^{I}$ \\
\hline \multicolumn{5}{|c|}{ Maximum amount of load transferred (in cubic meters) } \\
\hline
\end{tabular}

Step 4. Find the key element:

Min (max element)

$=$ Minimum of maximum element in each column

$=\min \left(\widetilde{0.0125}^{I},{\widetilde{0.0187}^{I}}^{I}=\widetilde{0.0125}^{I}\right.$

The numbers are compared using score function given in this paper.

Now $\widetilde{0.0125}^{I}$ is the key element or pivot element.

The row corresponding to the key element (first row) is the key row and the column corresponding to the key element (first column) is the key column.

Step 5. Rearrange the system based on pivot element:

$$
\begin{gathered}
\widetilde{c}_{j}^{I} \\
A \\
C
\end{gathered}\left(\begin{array}{ccc}
\tilde{x}_{1}^{I} & \tilde{x}_{2}^{I} & \\
\widetilde{24}^{I} & \widetilde{24}^{I} & \\
\widetilde{0.0125}^{I} & \widetilde{0.0094}^{I} & \tilde{1}^{I} \\
\widetilde{0.01}^{I} & \widetilde{0.015}^{I} & \tilde{1}^{I} \\
\widetilde{0.0042}^{I} & \widetilde{0.0187}^{I} & \tilde{1}^{I}
\end{array}\right) .
$$

Step 6. Consider the $2 \times 2$ system of equations:

$$
\begin{aligned}
\widetilde{0.0125}^{I} \tilde{x}_{1 e}^{I}+\widetilde{0.0094}^{I} \tilde{x}_{2 e}^{I} & =\tilde{1}^{I}, \\
\widetilde{0.01}^{I} \tilde{x}_{1 e}^{I}+\widetilde{0.015}^{I} \tilde{x}_{2 e}^{I} & =\tilde{1}^{I} .
\end{aligned}
$$

Solving these two equations we get the following:

Equalized profit units:

$$
\begin{aligned}
& \tilde{x}_{1 e}^{I}=\widetilde{59.68}^{I}, \\
& \tilde{x}_{2 e}^{I}=\widetilde{26.88}^{I} .
\end{aligned}
$$

Actual units produced:

$$
\begin{aligned}
& \tilde{x}_{1 e}^{I}=\widetilde{59.68}^{I}, \\
& \tilde{x}_{2 e}^{I}=1.5 \times \widetilde{26.88}^{I}=\widetilde{40.32}^{I},
\end{aligned}
$$

where $\widetilde{59.68}^{I}=\{(20.47,59.68,158.14) ; \quad(-35.67,59.68$, $243.92)\}$ and $\widetilde{40.32}^{I}=\{(27.67,40.32,60.63) ;(15.68,40.32$, 127.42)\}.
This indicates that approximately $\widetilde{59.68}^{I} \approx \widetilde{60}^{I}$ articulated trucks and $\widetilde{40.32}^{I} \approx \widetilde{40}^{I}$ dump trucks can be chosen by the site engineer to move a maximum amount of $\widetilde{2080} I$ cubic meters.

Step 7. Set up a profit table.

This step interprets the results of the previous steps in table form (Table 1).

\section{Discussion and Conclusion}

This algorithm can be an aid in a wide area of decision making. Its usefulness covers a broad range of problems. A specific problem in which a number of different demands compete for limited amounts of resources will demonstrate this technique. The objective is to meet as many of these demands as possible so as to be most beneficial over all.

The matrix-analysis method will quickly provide an optimum solution and greatly reduce the number of steps in the classical simplex method. This method starts with the benefit that will bring the largest overall returns; the simplex method starts with the benefit that brings the largest unit profit. There will be cases where it is impractical or perhaps impossible to solve the matrix using matrix-analysis method. As shown by the sample problem, the matrix-analysis method of IFLPP can be used to aid management decisions. The solution obtained through this method gives complete operating and profit information for management use. It is a formal, logical approach for making the best decision when alternatives and choices exist. In future work, the efficiency of this process can be greatly improved by detecting and removing redundant inequalities.

\section{Conflict of Interests}

The authors declare that there are no conflict of interests regarding the publication of this paper.

\section{Acknowledgments}

The authors would like to thank to the editor and anonymous referees for various suggestions which have led to an improvement in both the quality and clarity of the paper. 


\section{References}

[1] M. Wen and H. Li, "Fuzzy data envelopment analysis (DEA): model and ranking method," Journal of Computational and Applied Mathematics, vol. 223, no. 2, pp. 872-878, 2009.

[2] C. Kahraman, T. Ertay, and G. Büyüközkan, "A fuzzy optimization model for QFD planning process using analytic network approach," European Journal of Operational Research, vol. 171, no. 2, pp. 390-411, 2006.

[3] C. L. P. Chen, Y.-J. Liu, and G.-X. Wen, "Fuzzy neural networkbased adaptive control for a class of uncertain nonlinear stochastic systems," IEEE Transactions on Cybernetics, vol. 44, no. 5, pp. 583-593, 2014.

[4] Y.-J. Liu, S. Tong, and C. L. P. Chen, "Adaptive fuzzy control via observer design for uncertain nonlinear systems with unmodeled dynamics," IEEE Transactions on Fuzzy Systems, vol. 21, no. 2, pp. 275-288, 2013.

[5] W.-J. Wang and W.-W. Lin, "Decentralized PDC for large-scale T-S fuzzy systems," IEEE Transactions on Fuzzy Systems, vol. 13, no. 6, pp. 779-786, 2005.

[6] W.-J. Wang, Y.-J. Chen, and C.-H. Sun, "Relaxed stabilization criteria for discrete-time T-S fuzzy control systems based on a switching fuzzy model and piecewise Lyapunov function," IEEE Transactions on Systems, Man, and Cybernetics, Part B: Cybernetics, vol. 37, no. 3, pp. 551-559, 2007.

[7] S. Effati, M. Pakdaman, and M. Ranjbar, "A new fuzzy neural network model for solving fuzzy linear programming problems and its applications," Neural Computing and Applications, vol. 20, no. 8, pp. 1285-1294, 2011.

[8] M. Delgado, J. L. Verdegay, and M. A. Vila, "Fuzzy linear programming from classical methods to new applications," in Fuzzy Optimization. Recent Advances, M. Delgado, J. Kacprzyk, J. L. Verdegay, and M. A. Vila, Eds., pp. 111-134, Physica, 1994.

[9] K. T. Atanassov, "Intuitionistic fuzzy sets," Fuzzy Sets and Systems, vol. 20, no. 1, pp. 87-96, 1986.

[10] K. Atanassov and G. Gargov, "Interval valued intuitionistic fuzzy sets," Fuzzy Sets and Systems, vol. 31, no. 3, pp. 343-349, 1989.

[11] G. S. Mahapatra and T. K. Roy, "Intuitionistic fuzzy number and its arithmetic operation with application on system failure," Journal of Uncertain Systems, vol. 7, no. 2, pp. 92-107, 2013.

[12] J.-Q. Wang, R. Nie, H.-Y. Zhang, and X.-H. Chen, "New operators on triangular intuitionistic fuzzy numbers and their applications in system fault analysis," Information Sciences, vol. 251, pp. 79-95, 2013.

[13] D.-F. Li, "A ratio ranking method of triangular intuitionistic fuzzy numbers and its application to MADM problems," Computers and Mathematics with Applications, vol. 60, no. 6, pp. 1557-1570, 2010.

[14] J. Wu and F. Chiclana, "A risk attitudinal ranking method for interval-valued intuitionistic fuzzy numbers based on novel attitudinal expected score and accuracy functions," Applied Soft Computing, vol. 22, pp. 272-286, 2014.

[15] S.-P. Wan and J.-Y. Dong, "Possibility method for triangular intuitionistic fuzzy multi-attribute group decision making with incomplete weight information," International Journal of Computational Intelligence Systems, vol. 7, no. 1, pp. 65-79, 2014.

[16] L. Anzilli, G. Facchinetti, and G. Mastroleo, "Evaluation of ranking of intuitionistic fuzzy quantities," in Fuzzy Logic \& Applications, vol. 8256, pp. 139-149, Springer, 2013.
[17] H. Tanaka, T. Okuda, and K. Asai, "On fuzzy-mathematical programming," Journal of Cybernetics, vol. 3, no. 4, pp. 37-46, 1973.

[18] R. E. Bellman and L. A. Zadeh, "Decision-making in a fuzzy environment," Management Science, vol. 17, no. 4, pp. B141-B164, 1970.

[19] H.-J. Zimmermann, "Fuzzy programming and linear programming with several objective functions," Fuzzy Sets and Systems, vol. 1, no. 1, pp. 45-55, 1978.

[20] H.-J. Zimmerman, "Using fuzzy sets in operational research," European Journal of Operational Research, vol. 13, no. 3, pp. 201216, 1983.

[21] P. P. Angelov, "Optimization in an intuitionistic fuzzy environment," Fuzzy Sets and Systems, vol. 86, no. 3, pp. 299-306, 1997.

[22] B. Zhu and Z. Xu, "A fuzzy linear programming method for group decision making with additive reciprocal fuzzy preference relations," Fuzzy Sets and Systems, vol. 246, pp. 19-33, 2014.

[23] A. Mohtashami, "The optimal solution for several different degrees of feasibility for fuzzy linear and non-linear programming problems," Journal of Intelligent \& Fuzzy Systems, vol. 27, no. 5, pp. 2611-2622, 2014.

[24] A. K. Nishad and S. R. Singh, "Solving multi-objective decision making problem in intuitionistic fuzzy environment," International Journal of System Assurance Engineering and Management, vol. 6, no. 2, pp. 206-215, 2015.

[25] J. Ye, "A linear programming method based on an improved score function for interval-valued intuitionistic fuzzy multicriteria decision making," The Engineering Economist, vol. 58, no. 3, pp. 179-188, 2013.

[26] D.-F. Li, "Linear programming method for MADM with interval-valued intuitionistic fuzzy sets," Expert Systems with Applications, vol. 37, no. 8, pp. 5939-5945, 2010. 


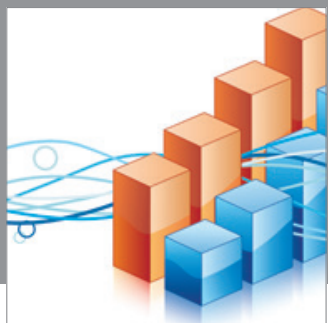

Advances in

Operations Research

mansans

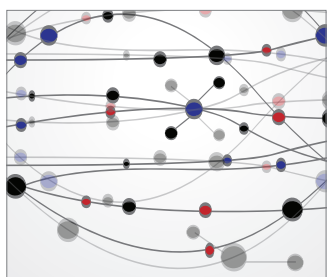

The Scientific World Journal
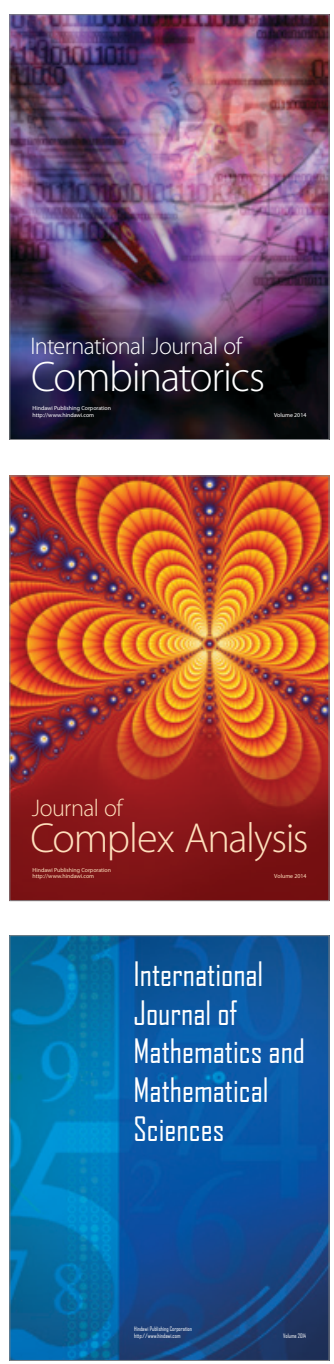
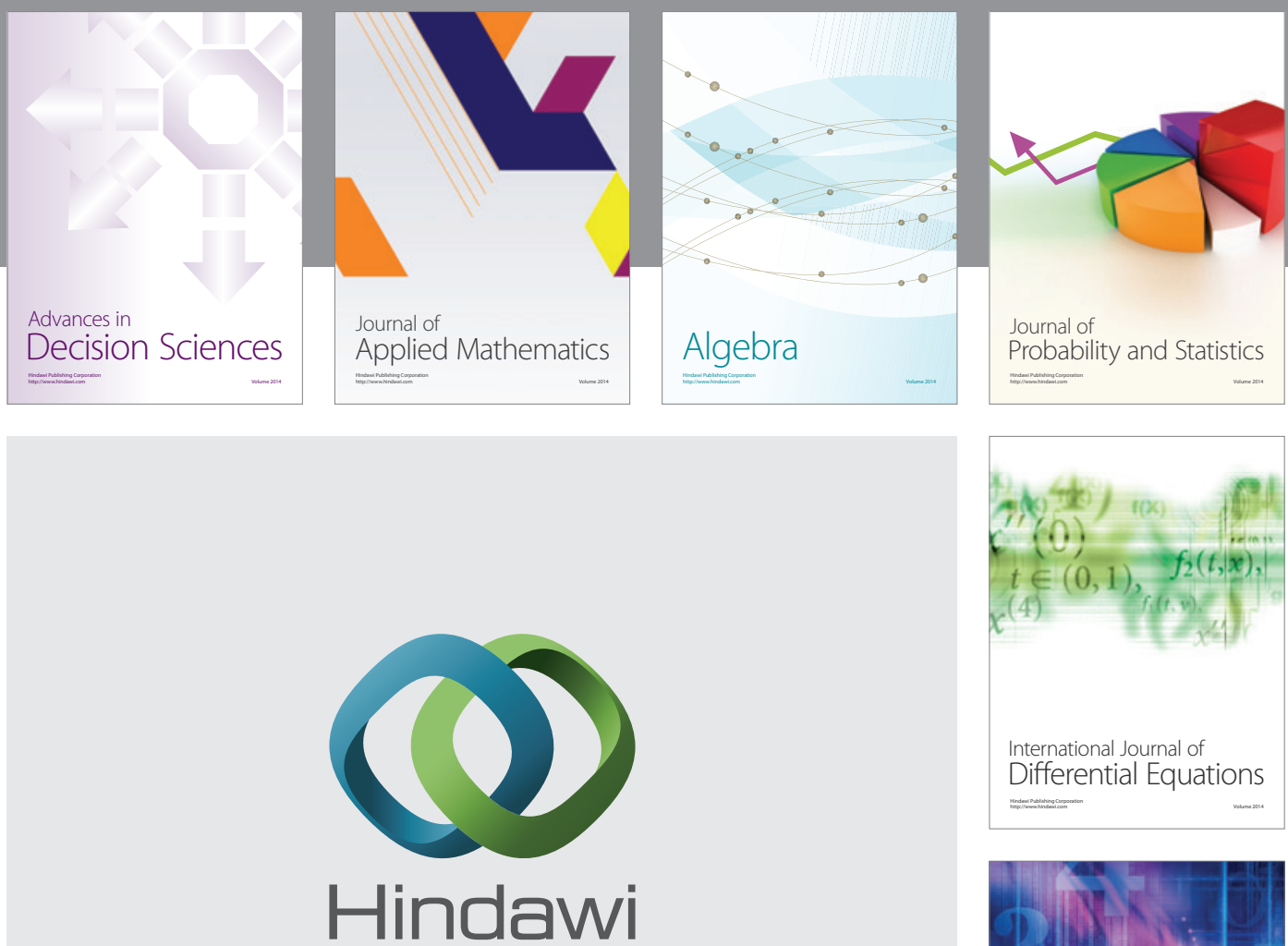

Submit your manuscripts at http://www.hindawi.com
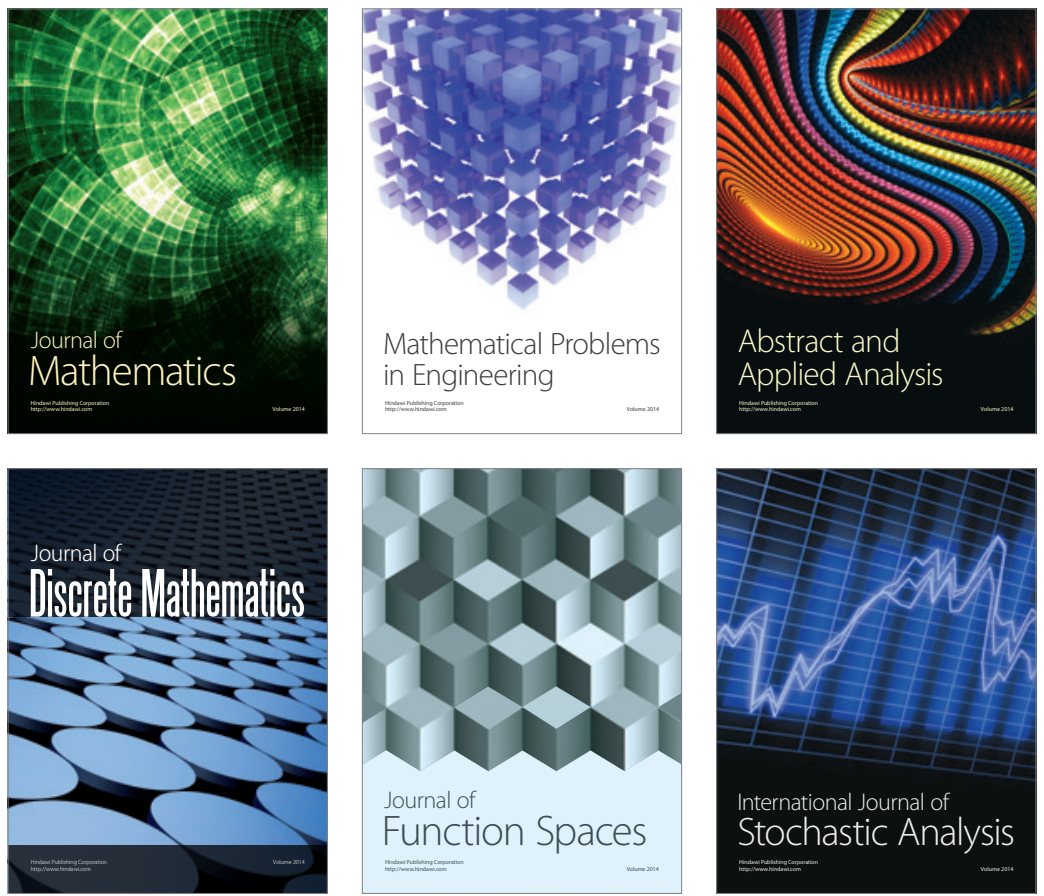

Journal of

Function Spaces

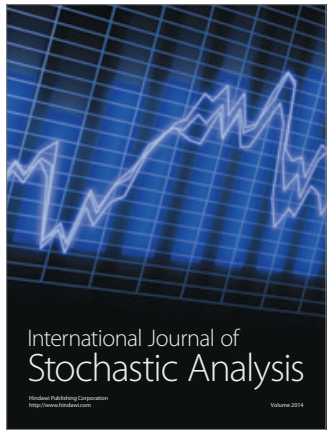

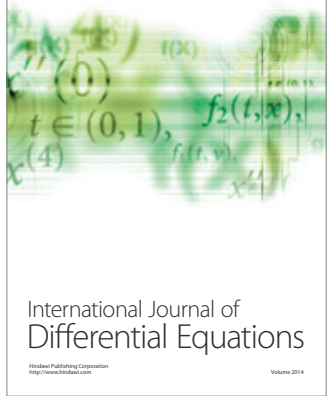
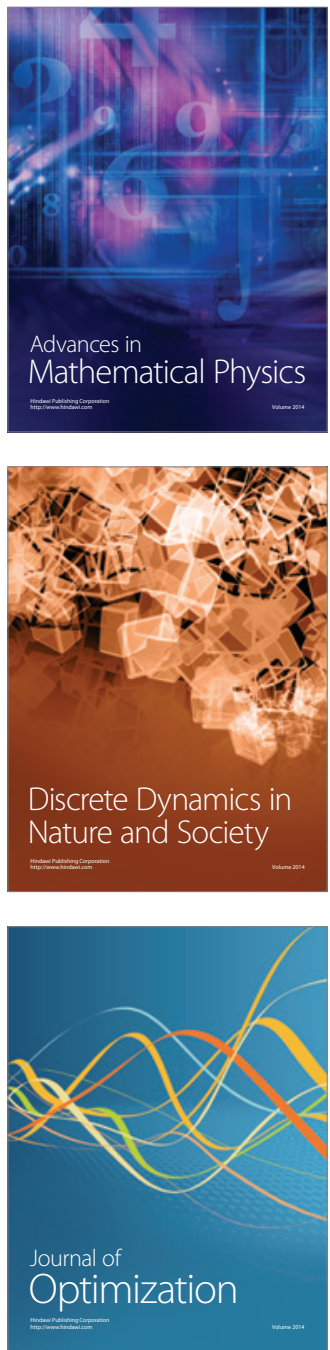